\title{
Charge fluctuations in the slave fermion representation of the $t-J$ model-evidence for phase separation from loop corrections
}

\author{
J W Rasul and Yujong Bai \\ Physics Department, University of Michigan, Ann Arbor, MI 48109-1120, USA
}

Received 14 March 1995, in final form 30 January 1996

\begin{abstract}
We investigate the charge density correlation function in the slave fermion representation of the two-dimensional $t-J$ model using the self-consistent perturbation approach developed for that model by Li et al (1992 Phys. Rev. B 45 5428), which to lowest order in $(t, J)$ gives a diagrammatic equivalent to the usual mean field theory. At the lowest order the equal-time correlation function (interpreted as the holon density correlation function) shows similar features to the results of recent high-temperature series calculations. However the loop corrections, representing scattering of holes by the antiferromagnetic spin fluctuations, lead to a divergent compressibility at low temperatures for low hole dopings $(\delta)$ and small $J / t$, which we interpret as indicating a tendency towards phase separation at a temperature $t^{2} \delta / J$.
\end{abstract}

\section{Introduction}

The question of charge correlations in low-dimensional strongly correlated electron systems and their relation to the underlying spin degrees of freedom remains a central issue in condensed matter physics. On the one hand the Luttinger liquid scenario [1] suggests separation of spin and charge in momentum space with, as some recent numerical results [2] suggest, separate Fermi surfaces. On the other hand a large section of the community favours spin and charge separation in real space, the so-called phase separation viewpoint [3-5] where charge carriers congregate in the same region of space so as to lower their kinetic energy.

Analytic formulations that separate out spin and charge explicitly have been invoked ever since the heavy-fermion systems were discovered. One of these, the slave boson [6] approach, treats the spins as fermionic and the charge as bosonic and generally leads to a Fermi liquid ground state in simple treatments, although some careful analyses of single and multiband models of high- $T_{c}$ systems $[7,8]$ do show phase separation at least at the mean field level. One of the present authors has shown that by going beyond mean field level as far as the two-loop level (order $1 / N^{2}$ ) that some aspects of Luttinger liquid behaviour do appear in the single-band $t-J$ model [9] if treated within this approach.

On the other hand, approaches to the quantum antiferromagnet have led to a rather different point of attack. It was shown by Arovas and Auerbach [10] that the 'Schwinger boson' representation of spin operators by combinations of boson operators $S_{i}=1 / 2 b_{i \sigma}^{+} \sigma b_{i \sigma}$, again with the appropriate local constraints enforced by Lagrange multipliers, when described in a mean field manner, could describe the spin liquid nature of the 1D Heisenberg model. In particular, the specific heat and low-temperature magnetization 
could be reconciled with the antiferromagnetic spin wave spectrum. Good qualitative results for both antiferromagnetic and ferromagnetic couplings (when compared with exact Bethe ansatz results) were obtained.

The good agreement in the 1D problem for the Schwinger boson representation of spins led other authors to explore the extension to the $2 \mathrm{D}$ doped antiferromagnet. This requires including a spinless fermion operator $\left(f_{i}\right)$ to label the charged 'hole' degree of freedom, i.e.,

$$
C_{i \sigma} \rightarrow f_{i}^{+} b_{i \sigma}
$$

again enforcing the constraint that each site be occupied either by a spin or by a hole. A number of authors [11-13] have studied the mean field theory of this model, concluding that the AF order in the half-field state is distorted by doping to yield an incommensurate ordering or 'spiral' phase. The 'inverse pitch' or incommensurate wavevector of this state varies proportionally with doping, although for larger dopings a ferromagnetic phase is found to be stable. Although earlier works were concerned with the nature of the spin ordering in such systems, it is also interesting to consider the charge degree of freedom in this 'slave fermion' representation.

The hole doping is then constrained to be equal to the number of holes, so that the holons have a Fermi surface identical to that of spinless electrons with density $\delta$. It should be stressed however that the holons themselves are not the physical electrons seen for example in a photoemission experiment. These require calculation of the convolution of holon and spinon-in fact mean field treatments [14] give electron Fermi surfaces centred on the incommensurate wavevector, while more careful analysis shows the transition, as the hole doping is increased, to a conventional electron-like Fermi surface [15]. Nevertheless, it should be remembered that the mean field slave fermion method itself has a problem reproducing the large Fermi surfaces seen in photoemission experiments—for such purposes the slave boson method is better suited. More recent work on this issue [16] has focused on which slave representation is better for the case of infinite $U$. A comparison of the ground state energies obtained (for various lattices) by the slave boson and slave fermion approaches with numerical results suggest that the slave boson representation is better for hole dopings larger than one-third-otherwise the slave fermion representation is better.

Our particular interest in the charge fluctuation spectrum of the slave fermion representation of the $t-J$ model stems from recent numerical work on the $2 \mathrm{D} t-J$ model, in which the high-temperature series expansion method was applied [2]. This method, in which correlation functions are expanded in 'cumulants', allows for expansion to fairly high orders in $[t, J] /$ temperature. The low-temperature limit is taken by applying Padé approximants to resum the series. The method has a long history in statistical mechanics, being able to compute critical exponents near phase transitions to excellent accuracy. The interesting result of this method for the $t-J$ model lies in the charge susceptibility at equal times $\langle\Delta n(k, 0) \Delta n(-k, 0)\rangle$. Normally this correlation function shows a peak at a value of $k$ corresponding, for non-interacting electrons, to twice the Fermi surface wavevector of the spin- $\frac{1}{2}$ non-interacting electrons $\left(2 k_{F}\right)$. In the strongly correlated $t-J$ model this peak is found to be twice the Fermi surface wavevector for spinless non-interacting electrons.

This is a new and surprising result. Our aim in this paper therefore is to reexamine the slave fermion $t-J$ model in which the charge degrees of freedom naturally correspond to spinless holons. Following the work of Li et al [17] we treat the single-particle propagators using a self-consistent perturbation theory in the hopping rate and exchange parameter which reproduces the saddle point results of earlier works. This approach has the advantage of allowing loop corrections to be evaluated diagrammatically. 
We start by interpreting the charge fluctuations as being due to the propagation of holon density operators. We then examine the charge correlation function using perturbation theory in $t$ (valid for $t \gg J$ ) in terms of the fermion propagators calculated at the self-consistent level. We find the fermions experience an interaction at this order mediated by the exchange of pairs of Schwinger bosons. As far as the charge susceptibility is concerned, whereas the mean field level reproduces qualitatively the results of Puttika et al [2], we find that dressing the fermion loops entering the charge susceptibility with ladder and bubble diagrams to all orders in the boson induced fermion-fermion interaction leads to an instability at low wavevectors and energies.

We interpret this instability as denoting a tendency towards phase separation-although such conclusions have in fact been reached before by treating the slave fermion model at mean field level $[19,20]$, we are able to provide a mechanism for such an instability arising from the exchange of antiferromagnetic fluctuations. The plan of the paper is as follows: in section 2 we recap on the self-consistent theory and in section 3 we discuss the lowestorder charge susceptibility. In section 4 we include the loop corrections and we present the conclusions and place the work in the context of other studies in section 5 .

\section{Self-consistent perturbation theory}

The $t-J$ model Hamiltonian which is equivalent to the large- $U\left(J=4 t^{2} / U\right)$ Hubbard model includes hopping $(t)$ of electrons between neighbouring sites and a spin-exchange $(J)$ interaction.

$$
H=-t \sum_{\langle i j\rangle} \sum_{\sigma} C_{i \sigma}^{+} C_{j \sigma}+\mathrm{HC}+J \sum_{\langle i j\rangle}\left(S_{i} S_{j}-\frac{1}{4} n_{i} n_{j}\right)
$$

where $C_{i \sigma}^{+}$is the creation operator of an electron with spin $\sigma$ or at site $i$ and the sum is over nearest-neighbour sites. Due to the strong Coulomb repulsion between electrons with opposite spins at the same site, we need to impose the single-occupancy constraint $\sum_{\sigma} C_{i \sigma}^{+} C_{i \sigma}=1$ or 0 . Then the effective Hamiltonian is written explicitly in terms of spin-pair operators $B_{i j}^{+}$defined as

$$
B_{i j}^{+}=\frac{1}{\sqrt{2}}\left(b_{i \uparrow}^{+} b_{j \downarrow}^{+}-b_{i \downarrow}^{+} b_{j \uparrow}^{+}\right)
$$

for singlet states. In addition, we represent the electron as a composite particle of (fermion) hole times (boson) spin. Then the on-site constraint

$$
\sum_{\sigma} b_{i \sigma}^{+} b_{i \sigma}+f_{i}^{+} f_{i}=1
$$

is imposed on average, with $\sum_{i} f_{i}^{+} f_{i}=\delta N$ ( $\delta$ is the hole doping concentration) incorporated into the Hamiltonian. The electron sum rule

$$
\sum_{\sigma} C_{i \sigma}^{+} C_{i \sigma}=1-\delta
$$

is then satisfied as well.

Now the Hamiltonian in the singly occupied subspace is given in terms of these $f$ (hole) and $b$ (spin) operators

$$
\begin{gathered}
H=-t \sum_{\langle i j\rangle} \sum_{\sigma}\left[f_{i} f_{j}^{+} b_{i \sigma}^{+} b_{j \sigma}+\mathrm{HC}\right]-J \sum_{\langle i j\rangle} B_{i j}^{+} B_{i j}-\mu_{F} \sum_{i} f_{i}^{+} f_{i} \\
-\mu_{B} \sum_{i \sigma}\left(b_{i \sigma}^{+} b_{i \sigma}+f_{i}^{+} f_{i}-1\right) .
\end{gathered}
$$


The Lagrange multiplier terms $\mu_{F}$ and $\mu_{B}$ are introduced to minimize the averaged Hamiltonian. The (fictitious) chemical potentials $\mu_{B}$ and $\mu_{F}$ are treated as fixed, until we determine them in the end from the condition that the total number of particles is fixed. To study the spin-spin and hole-spin correlations, we need to define appropriate propagators in perturbation expansions. These expansions are then performed in powers of the hopping rate and exchange parameter [17]. Since the Schwinger boson number is known not to be strictly conserved in the mean field treatments of the $t-J$ model we have to allow for the existence of 'anomalous propagators' which create or destroy anti-parallel spin pairs.

We work explicitly with finite-temperature expressions i.e. using the Matsubara formalism in which the averages are taken over a grand canonical ensemble. The anomalous (spin-boson) propagators are defined as $F(k, \tau)=-\left\langle T_{\tau} b_{-k \downarrow}^{+}(\tau) b_{k \uparrow}^{+}(0)\right\rangle . \quad F(k, \tau)$ is the probability amplitude of creating a pair of spins with opposite momenta and spin states. It can be shown that $F(k, 0)$ is proportional to the energy gap of the spin excitation spectrum. We also need to introduce the normal propagator $G\left(k_{\uparrow}, \tau\right)=-\left\langle T_{\tau} b_{k \uparrow}(\tau) b_{k \uparrow}^{+}(0)\right\rangle$.

Substituting each normal propagator in perturbation expansions by the generalized one, we obtain valid propagators for the states undergoing phase transitions. The structure of self-consistent perturbation theory is maintained using Dyson's equation, written in terms of the full matrix propagators. The boson self-energy in this generalized formulation above is calculated to first order in the hopping and exchange parts of the Hamiltonian, and represents the internal spin-aligning field (the diagonal elements) as well as the spin-pairing field (the off-diagonal elements).

Next we transform the hole-fermion $f_{i}$ and spin-boson $b_{i \sigma}$ operators to momentum space on a square lattice with lattice constant $a$. We have then to introduce the 'form factor' $\gamma(p)=\cos \left(p_{x}\right)+\cos \left(p_{y}\right)$ in terms of which the hopping part of the interaction becomes

$$
H_{t}=-\frac{2 t}{N^{2}} \sum_{k, p, q} \sum_{\sigma} \gamma(k-p) f_{k} f_{k-q}^{+} b_{p \sigma}^{+} b_{p-q, \sigma}+\mathrm{HC} .
$$

Now we compute the contribution of the $H_{t}$ part to the self-energy of the boson. The first-order self-energy diagram for the boson propagator is the dressed fermion loop. We introduce the order parameter $\phi=\frac{1}{N} \sum_{k} \gamma(k) \frac{1}{\beta} \sum_{\omega_{n}} G_{f}\left(k, \omega_{n}\right)=\left\langle f_{i}^{+} f_{j}\right\rangle$ which is a holehopping amplitude or charge-bonding parameter, since the hole is the charge carrier in this representation (a non-zero value of $\phi$ implies hole mobility). Then, the self-energy is written in terms of this as (for the diagonal elements) $\sum_{b b^{+}}(p, \omega)=-t \gamma(p) \phi$.

As with $H_{t}$, we can also carry out self-consistent first-order perturbation theory in $H_{J}$. The difference this time is that we have to consider off-diagonal as well as diagonal self-energies resulting from spin-pairing interaction. The self-consistent approach has the advantage that dynamical corrections can be systematically calculated, as we shall we in the next section.

Transforming $H_{J}$ to momentum space we obtain

$$
H_{J}=-J \sum_{k p q} \gamma_{c}(k-p)\left(b_{k}^{+} b_{-k-q}^{+}-b_{k}^{+} b_{-k-q}^{+}\right)\left(b_{p} b_{-p+q}-b_{p} b_{-p+q}\right) \text {. }
$$

Then the off-diagonal self-energy becomes $\sum_{b^{+} b^{+}}(k)=J \gamma_{s}(k) \Delta$ where $\gamma_{s}(k)=\sin \left(k_{x}\right)-$ $\sin \left(k_{y}\right)$ and the spin-pairing order parameter is defined as $\Delta=(1 / 2 \beta) \sum_{p \omega_{n}} \gamma_{s}(p) F\left(p, \omega_{n}\right)$ with a similar expression for the conjugate self-energy. The diagonal part of the boson self-energy can likewise be obtained as $\sum_{b b^{+}}=-J \gamma_{c}(p) \chi / 2$ with the spinhopping order parameter (arising from the exchange term alone) defined by $\chi=$ $(1 / 2 \beta) \sum_{p, \omega_{n}} \gamma_{c}(p) G\left(p, \omega_{n}\right)$. 
Defining $Q(p)=\left(t \phi-\frac{J}{2} \chi\right) \gamma_{c}(p)$, we obtain the diagonal elements of the inverse Bose propagator as $-\omega-\mu_{B}+Q(p)$ while the off-diagonal elements of the inverse Bose propagator are simply $J \gamma_{s}(p) \Delta$. One can simply derive the dispersion relation for the spin excitations from the poles of the matrix propagator as [15]

$$
\omega^{2}=\left(\mu_{B}-Q(p)\right)^{2}-J^{2} \gamma_{s}(p)^{2} \Delta^{2}
$$

which shows a gap on the scale of $J$. The minimum of $\omega$ decreases as the temperature approaches zero. The zero mode is obtained at $\omega_{0}=\sqrt{\left(t \phi-\frac{J}{2} \chi\right)^{2}+(J \Delta)^{2}}$ at wavevectors $k_{0}$ with $\cos k_{0}=-\left(t \phi-\frac{J}{2} \chi\right) / \omega_{0}$. At $k_{0}=0$, the system is in a ferromagnetic phase, while at $k_{0}=\frac{\pi}{2}$ it is antiferromagnetic. The quantities $\phi, \chi$ and $\Delta$ representing the hole hopping rate, the ferromagnetic order parameter and antiferromagnetic order parameter respectively are then determined self-consistently. These self-consistent relations are identical to the results of mean field theory, which have been discussed in a number of papers $[11,12,17]$. In the undoped limit $(\delta=0)$, the spin and holon hopping rates are zero, and $\Delta$ is at its maximum value. Both $\chi$ and $\phi$ increase approximately linearly with $\delta$ increasing while $\Delta$ decreases and vanishes for $\delta=J / t$. At $T=0$ (for 2D) there is Bose condensation of spins into a spiral spin state with long-range order. At finite temperature, the spins show short-range incommensurate antiferromagnetic correlations for small dopings. At dopings larger than $\delta \sim J / t$, the ordering tendencies become ferromagnetic. We will return to a more detailed examination of the mean field equations later.

As with the boson propagator, we now calculate the hole-fermion Green function by first computing its self-energy. The first-order diagram for the (hole) self-energy is the boson loop with the interaction from above $H_{t}$ at the vertex. Then the self-energy of the hole reads

$$
\Sigma_{f}^{t}(k)=\frac{-2 t}{N^{2}} \sum_{p} \gamma(k-p) \frac{1}{\beta} \sum_{\omega_{n}}\left[G_{b}\left(p, \omega_{n}\right)_{(11)}+G_{b}\left(p, \omega_{n}\right)_{(22)}\right]
$$

where the diagonal matrix elements of the Bose propagator, i.e. $G_{b}\left(p, \omega_{n}\right)$ are given above. Employing the spin-hopping OP we had earlier the self-energy written $\Sigma_{f}(k)=-2 t \chi \gamma_{c}(k)$ which shows a tight-binding dispersion relation, coming from the poles of the fermion Green function

$$
G_{f}(k, \omega)=1 /\left[\omega+\mu_{B}+2 t \chi \gamma_{c}(k)\right]
$$

The chemical potential is determined from the condition $\sum_{k, \omega} G_{f}(k, \omega)=\delta$ which determines the size of the holon Fermi surface. Qualitatively, the holon Fermi wavevector $k_{F}$ scales as $\delta^{1 / 2}$ while the spin hopping rate is proportional to $\chi$ and linear in $1-\delta$. Thus, the holons are heavy with a mass enhancement $\sim 1 / \delta$ with a small Fermi surface. In fact this is only a part of the true charge response-both numerical results [18] and loop corrections to the holon self-energy [17] find that the true holon bandwidth is of order $t$. The above expression (11) only contains the coherent component of the holon propagator. However the approach used in this paper of summing up ladder and bubble contributions to the charge susceptibility only really treats the two-particle intermediate states in a reliable fashion. Furthermore, as in all such microscopic Landau-like approaches, it is only the coherent components of the intermediate propagators that yield the important singular low-energy and wavevector contributions to the two-particle intermediate states-therefore we are justified in retaining only the coherent mean field contribution (11) to the holon propagator. 


\section{Charge susceptibility—lowest order}

Our main interest in the $t-J$ model lies in the nature of the charge fluctuations (rather than the spin fluctuations which earlier works focused on). We therefore need to examine the charge correlation function

$$
\chi\{i, j, \tau\}=-\left\langle T_{\tau} n_{i}(\tau) n_{j}(0)\right\rangle
$$

where $n_{i}=\sum_{\sigma} C_{i \sigma}^{+} C_{i \sigma}$ is the charge density operator. In the slave fermion representation the charge density operator becomes

$$
n_{i}=\sum_{\sigma} b_{i \sigma}^{+} f_{i} f_{i}^{+} b_{i \sigma}
$$

which means that the correlation function $\chi$ involves a combination of eight operators. Therefore the method of calculation becomes crucial-a great simplification is made if the occupancy constraint is enforced, because in that case

$$
n_{i}=f_{i} f_{i}^{+}\left(1-f_{i}^{+} f_{i}\right)=1-f_{i}^{+} f_{i}=f_{i} f_{i}^{+}
$$

that is the electron density is one minus the hole density. At least at the mean field level, we know that the constraint is satisfied. Then the holon charge susceptibility becomes $\chi\{i, j, \tau\}=-\left\langle T_{\tau} f_{i} f_{i}^{+} f_{j} f_{j}^{+}\right\rangle$which in mean field theory is simply the Lindhard function for holes with the dispersion relation given by (12). After Fourier transforming, we can write

$$
\chi(q, \mathrm{i} \omega)=\sum_{k} \chi_{0}(k, q, \mathrm{i} \omega)=\sum_{k} \frac{f\left(\epsilon_{k+q}\right)-f\left(\epsilon_{k}\right)}{\left(\mathrm{i} \omega-\epsilon_{k+q}+\epsilon_{k}\right)}
$$

where $\epsilon_{k}=2 t \chi \gamma(k)=2 t \chi\left(\cos k_{x}+\cos k_{y}\right)$ is the holon dispersion and we have defined through this equation a subsidiary Lindhard function (not yet summed over wavevector) $\chi_{0}(k, q, \mathrm{i} \omega)$ which will prove useful later. Fourier transforming in frequency we obtain the equal-time correlation function as

$\chi(q, \tau=0)=\sum_{k} n\left(\epsilon_{k+q}-\epsilon_{k}\right)\left[f\left(\epsilon_{k+q}\right)-f\left(\epsilon_{k}\right)\right]=\sum_{k} f\left(\epsilon_{k}\right)\left(1-f\left(\epsilon_{k+q}\right)\right)$

where $n(\epsilon)$ is the Bose distribution function and $f(\epsilon)$ the hole Fermi function. Thus, we have that the charge density correlation function is one 'cap' of $k$-space trapped between two spinless holon Fermi surfaces displaced by wavevector $q$. We can see easily that this volume of $k$-space is identical to the volume inside the one cap produced by the overlapping electron Fermi function combination $\sum_{\sigma}\left(1-f\left(\epsilon_{i}\right)\right) f\left(\epsilon_{k+q}\right)$. Thus, we can see that for the $t-J$ model in the slave fermion representation, at the mean field level, the equal-time density correlation function is similar to that of spinless electrons with the same electron count. This type of correlation function is the same as that used by Puttika et al [2] to compare with high-temperature series results for the $t-J$ model. The difference however is that our holon energy is somewhat narrower than the bare spinless fermion energy used by those authors, since in our case the holon hopping rate is renormalized by the spinon hopping probability, which vanishes in the undoped limit. This changes the details of the temperature dependence of the equal-time correlation function, although the results become identical at zero temperature. This discrepancy reflects that fact mentioned earlier that the mean field results only capture part of the holon bandwidth found in numerical finite-size calculations [18] and in loop corrections to the holon self-energy [17]. Nevertheless we shall continue by examining scattering of the holons by the antiferromagnetic fluctuations and the consequences for the charge susceptibility. 
In going beyond the mean field level, the constraint used in defining $\chi(q, \tau)$ becomes harder to enforce. Such constraints are naturally enforced within functional integral techniques, and, since we have chosen the more physically intuitive diagrammatic method, we shall always have to bear in mind the possible dynamic effects arising from the boson operators. However, we will assume the charge density depends primarily on the actual propagation of holon density operators, and focus on one aspect of the charge density, namely the effective fermion-fermion interaction mediated by the antiferromagnetic spin bosons and only so far as it dresses the spinless fermionic Lindhard function.

\section{Charge susceptibility-loop corrections}

We calculate the loop corrections to the charge susceptibility of the electron using the diagrammatic method. We shall use the hopping term in the Hamiltonian to generate the particle-hole terms that we expect to be dominant at low frequencies and wavevectors. We shall sum therefore, then bubble and ladder diagrams that correspond, as in the electron gas and Hubbard models, to the dominant low- $\omega, q$ behaviour [19], using the hole-hole interaction generated by $H_{t}$ at order $t^{2}$. Our approach is then consistent with assuming a weak interaction. The antiferromagnetic interaction $J$ dresses this hole-hole interaction (and may be ignored for $t \gg J$ ). We shall only include antiferromagnetic interactions through their one-loop self-consistent renormalizations of the boson propagators described in the previous section. Thus the intermediate boson and holon propagators are those obtained at the mean field level. The bubble contributions to the charge susceptibility series are given as follows. The notation for the total sum of the bubble series (see figure 1) is

$$
\frac{1}{\beta^{2}} \sum_{k_{1}, \omega_{1}} \sum_{p_{1}, v_{1}} \chi_{0}\left(k_{1}, q, v_{q}\right) Y\left(k_{2}, v_{q}, q\right)
$$

that is, we label

$Y\left(k_{2} v_{1}, q\right)=1+\frac{1}{\beta} \sum_{k_{3}} V_{e f f}\left(k_{2}, q, k_{3}, v_{q}\right) \frac{1}{\beta} \sum_{\omega_{3}} \chi_{0}\left(k_{3}, q, \omega_{3}, v_{q}\right) Y\left(k_{3}, v_{q}, q\right)$

where the holon-holon interaction is defined by (see figure 2)

$$
\begin{gathered}
V_{e f f}\left(k_{1}, q, k_{2}, v_{q}\right)=\sum_{p} \gamma\left(k_{1}-p_{1}\right) \frac{1}{\beta} \sum_{v_{1}}\left\{V_{d}\left(p_{1}, q, v_{q}, v_{1}\right) \gamma\left(p_{1}-k_{2}\right)\right. \\
\left.+V_{o d}\left(p_{1}, q, v_{q}, v_{1}\right) \gamma\left(k_{2}+p_{1}+q\right)\right\}
\end{gathered}
$$

and the factor of $-2 t / N$ is absorbed in $\gamma_{c}$. The function $\chi_{0}$ is the same subsidiary Lindhard function as defined in (22) while the quantities $V_{d}$ and $V_{o d}$ are the diagonal and off-diagonal boson bubbles respectively. This series has formed similarities to the usual RPA approach, the only complication being the momentum dependence of the vertices which leads us to deal with integral equations instead of a single geometric sum. Despite its formal difficulty, we will later show that for small hole doping the series simplifies considerably. To exact fully low- $\omega, q$ behaviour in the integral equation, we need to isolate the low- $\omega, q$ limit of the effective interaction. In order to calculate the effective interaction, we first compute the diagonal boson bubble $V_{d}$.

$$
\frac{1}{\beta} \sum_{v_{1}} V_{d}\left(p_{1}, q, v_{q}, v_{1}\right)=\frac{1}{\beta} \sum_{v_{1}} G_{b}\left(p_{1}+q, v_{1}+v_{q}\right) G_{b}\left(p_{1}, v_{1}\right)
$$




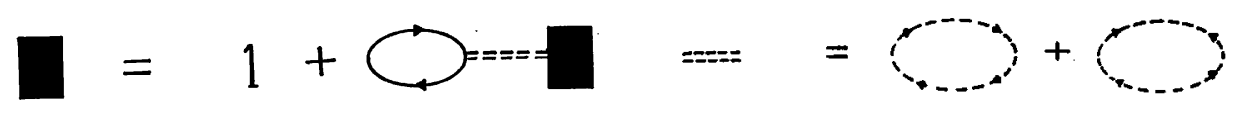

Figure 1. Bubble series for the charge susceptibility defined in (18). The single lines denote the holon propagators while the double dotted line denotes the

Figure 2. The actual diagonal and off-diagonal elements of the double-boson exchange that mediates an interaction between holons. effective holon-holon interaction defined in (19).

We convert the sum over the boson frequency, using the Bose function $N(\omega)$, and convert to an integral around a contour of a large semicircle (clockwise) excluding the poles of $N(\omega)$.

$$
\begin{aligned}
\frac{1}{\beta} \sum_{v_{1}} V_{d}\left(p_{1}, q\right. & \left., v_{q}, v_{1}\right)=\left\{\frac{N\left(-\omega_{p_{1}+q}\right)\left(\omega_{p_{1}+q}-\mu+Q_{p_{1}+q}\right)\left(\mathrm{i} v_{q}+\omega_{p_{1}+q}-\mu+Q_{p_{1}}\right)}{2 \omega_{p_{1}+q}\left(\mathrm{i} v_{q}+\omega_{p_{1}+q}-\omega_{p_{1}}\right)\left(\mathrm{i} v_{q}+\omega_{p_{1}+q}+\omega_{p_{1}}\right)}\right. \\
& +\frac{N\left(-\omega_{p_{1}}\right)\left(\omega_{p_{1}}-\mu+Q_{p_{1}+q}\right)\left(\omega_{p_{1}}-\mathrm{i} v_{q}-\mu+Q_{p_{1}}\right)}{2 \omega_{p_{1}}\left(\omega_{p_{1}}-\mathrm{i} v_{q}-\omega_{p_{1}+q}\right)\left(\omega_{p_{1}+q}-\mathrm{i} v_{q}+\omega_{p_{1}}\right)} \\
& +\frac{N\left(\omega_{p_{1}+q}\right)\left(\omega_{p_{1}+q}+\mu-Q_{p_{1}+q}\right)\left(\mathrm{i} v_{q}-\omega_{p_{1}+q}-\mu+Q_{p_{1}}\right)}{2 \omega_{p_{1}+q}\left(\mathrm{i} v_{q}-\omega_{p_{1}+q}+\omega_{p_{1}}\right)} \\
& \left.+\frac{N\left(\omega_{p_{1}}\right)\left(\omega_{p_{1}}+\mu-Q_{p_{1}+q}\right)\left(\omega_{p_{1}}+\mathrm{i} v_{q}+\mu-Q_{p_{1}}\right)}{2 \omega_{p_{1}}\left(\omega_{p_{1}}+\mathrm{i} v_{q}+\omega_{p_{1}+q}\right)\left(\mathrm{i} v_{q}+\omega_{p_{1}}-\omega_{p_{1}+q}\right)}\right\} .
\end{aligned}
$$

Taking the zero-frequency limit of $v_{q}=0$ for the boson,

$\frac{1}{\beta} \sum_{v_{1}} V_{d}\left(p_{1}, q, v_{q}=0, v_{1}\right)=\frac{\left(\mu-Q\left(p_{1}\right)\right)\left(\mu-Q\left(p_{1}+q\right)\right)-\omega_{p_{1}} \omega_{p_{1}+q}}{2 \omega_{p_{1}} \omega_{p_{1}+q}\left(\omega_{p_{1}}+\omega_{p_{1}+q}\right)}$.

The off-diagonal component denoted as $V_{o d}$ is also similarly evaluated

$$
V_{o d}=\frac{1}{\beta} \sum F\left(p_{1}+q, v_{1}+v_{q}\right) F\left(p_{1}, v_{1}\right) .
$$

Using the same procedure as above to carry out the frequency summation, we obtain for $\omega=0, q=0$

$$
\begin{gathered}
\frac{1}{\beta} \sum_{v_{1}} V_{o d}\left(p_{1}, q=0, v_{q}=0, v_{1}\right)=\frac{1}{\beta} \sum_{v_{1}} V_{d}\left(p_{1}, q=0, v_{q}=0, v_{1}\right) \\
=\frac{J^{2}\left|\Delta_{p}\right|^{2}}{4 \omega_{p_{1}}^{3}} \operatorname{coth}\left(\beta \omega_{p_{1}} / 2\right)
\end{gathered}
$$

so the effective holon-holon interaction at zero $\omega, q$ takes the form

$V_{e f f}\left(k_{1}, k_{2}\right)=\sum_{p} \gamma\left(k_{1}-p\right)\left\{\gamma\left(p-k_{2}\right)+\gamma\left(p+k_{2}\right)\right\} \frac{J^{2}\left|\Delta_{p}\right|^{2}}{4 \omega_{p_{1}}^{3}} \operatorname{coth}\left(\beta \omega_{p_{1}} / 2\right)$.

Now to understand the low- $\omega, q$ limit, we have to set other wavevectors on the hole Fermi surface, so we should compare the hole Fermi surface wavevector to the Brillouin zone size. In the case of the doping holes being very small, we can estimate the hole wavevector $k_{F}(h)$ by assuming a spherical hole Fermi surface

$$
\delta=\sum_{k<k_{F}(h)}=\int_{0}^{k_{F}(h)} \mathrm{d} k \frac{k}{2 \pi}=\frac{k_{F}^{2}(h)}{4 \pi} .
$$

Hence, $k_{F}(h) \simeq \sqrt{4 \pi \delta}$ which is much smaller than the Brillouin zone. Therefore, we can ignore $k_{1}$ and $k_{2}$ in comparison with $p_{1}$, as far as the evaluation of $V_{e f f}$ is concerned. 
Then,

$$
V_{e f f}\left(k_{1}, k_{2}\right) \approx V_{e f f}=2 \sum_{p_{1}} \gamma^{2}\left(p_{1}\right) J^{2}\left|\Delta_{p_{1}}\right|^{2} \frac{\operatorname{coth}\left(\beta \omega_{p_{1} / 2}\right)}{4 \omega_{p_{1}}^{3}} .
$$

Now we turn to the ladder contributions to the charge susceptibility (see figure 3 ). The ladder interaction between holons takes a form which, after allowing for diagonal and off-diagonal pairings of the boson lines, yields a contribution

$$
\begin{aligned}
\tilde{V}^{l a d}\left(q, v_{q}, k_{1}\right. & \left.-k_{2}, \omega_{1}-\omega_{2}\right)=\frac{1}{\beta} \sum_{v_{p}, p} \gamma\left(k_{2}+q-p\right)\left\{G ( p , \epsilon _ { p } ) G \left(p+k_{1}-k_{2}, \epsilon_{p}\right.\right. \\
& \left.+\omega_{1}-\omega_{2}\right) \gamma\left(k_{2}-p\right)+F\left(p, \epsilon_{p}\right) F\left(p+k_{1}-k_{2}, \epsilon_{p}+\omega_{1}-\omega_{2}\right) \\
& \left.\times \gamma\left(2 k_{1}-k_{2}+p\right)\right\} .
\end{aligned}
$$

Our ultimate interest is in the $q, v_{q} \rightarrow 0$ limit of this interaction, which may be written in terms of the bubble interaction as

$V^{l a d}\left(0,0, k_{1}-k_{2}, \omega_{1}-\omega_{2}\right)=V^{e f f}\left(k_{1}, k_{2}, q=k_{1}-k_{1}, v_{q}=\omega_{1}-\omega_{2}\right)$.

Meanwhile, the full ladder series for the holon bubble entering the charge susceptibility becomes

$$
=\frac{1}{\beta} \sum_{k_{1}, \omega_{1}} \chi_{0}\left(k_{1}, q, \omega_{1}, v_{q}\right) Y\left(k_{1}, v_{q}, q\right)
$$

where the ladder sum is represented as an integral equation

$$
\begin{aligned}
\tilde{Y}\left(k_{1}, v_{q}, q\right)= & 1+\frac{1}{\beta} \sum_{k_{2}, \omega_{2}} V^{l a d}\left(q, v_{q}, k_{1}-k_{2}, \omega_{1}-\omega_{2}\right)(-1) \\
& \times \chi_{0}\left(k_{2}, q, \omega_{2}, v_{q}\right) \tilde{Y}\left(k_{2}, v_{q}, q\right)
\end{aligned}
$$

which in the low-frequency, low-wavevector limit takes the form

$\tilde{Y}\left(k_{1}\right)=1-\frac{1}{\beta} \sum_{k_{2}, \omega_{2}} V_{e f f}\left(k_{1}, k_{2}, \omega_{1}-\omega_{2}, k_{2}-k_{1}\right) \chi_{0}\left(k_{2}, q, \omega_{2}, v_{q}\right) \tilde{Y}\left(k_{2}\right)$

written now in terms of the effective bubble diagram interaction. While for general dopings we have to include the full momentum dependence of the interaction, we note again that, as in the bubble series, the analysis simplifies considerably for small fillings. It may easily be shown that the intermediate frequency sums yield Fermi functions, which for small $q$ fix the energies in $V_{e f f}$ within the narrow coherent holon band, and fix the wavevectors on the small-holon Fermi surface. It follows that, as in the bubble case, the energy and wavevector dependence of the effective interaction may be dropped for small hole dopings, since the wavevector sum in (33) is over the entire Brillouin zone. We obtain a closed expression for $\tilde{Y}$

$$
\tilde{Y}=1 /\left[1-V_{e f f} \chi\left(q, \mathrm{i} v_{q}\right)\right] .
$$

Combining this expression with the bubble sum vertex,

$$
Y=1 /\left[1+V_{\text {eff }} \chi\left(q, \mathrm{i} v_{q}\right)\right]
$$

and subtracting the leading-order bubble (which we have double counted in both series), we obtain for the charge susceptibility from both ladder and bubble series,

$$
\begin{aligned}
\chi\left(q, \mathrm{i} v_{q}\right)= & \chi(0,0)\{\tilde{Y}+Y-1\} \\
& =\chi(0,0)\left[1+\left(\tilde{V}_{e f f} \chi\left(q, \mathrm{i} v_{q}\right)\right)^{2}\right] /\left[1-\left(\tilde{V}_{e f f} \chi\left(q, \mathrm{i} v_{q}\right)\right)^{2}\right]
\end{aligned}
$$




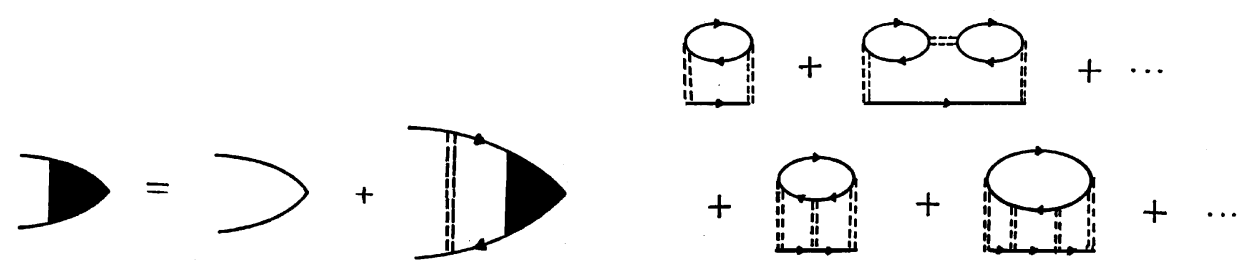

Figure 3. Ladder series for the charge susceptibility defined in (26). The symbols are the same as in figure 1 .

Figure 4. Ladder and bubble sums that enter into the effective interaction that yields loop corrections to the fermion self-energy.

which shows a divergence at the point where $\left|\tilde{V}_{e f f} \chi\left(q, \mathrm{i} v_{q}\right)\right|=1$, showing an instability presumably against charge ordering in the ground state given by the mean field isolation.

We can check the consistency of our diagrammatic result against the usual weak-coupling approach of summing ladder and bubble diagrams as in for example the Hubbard model [19] treated within the paramagnon approach. Once we allow for the spinless nature of the fermions, which allows both even and odd numbers of fermion loops in the bubble graphs, it can be straightforwardly shown (see figure 4) that the full fermion self-energy (resulting from holon scattering off the antiferromagnetic fluctuations) is a convolution of the fermion propagator with the effective interaction $V_{\text {tot }}(q, \mathrm{i} \omega)=V_{\text {eff }}^{2} \chi(q, \mathrm{i} \omega)$.

Thus we obtain a pole in the density-density correlation function where $\left|V_{\text {eff }} \chi(q, \omega)\right|=$ 1 where the effective fermion-fermion interaction is, for finite temperatures, given by (26). We have already approximated this function by its zero-frequency, zero-wavevector limit, since the coherent holon Fermi wavevector and energy are much smaller than the zone boundary and the antiferromagnetic order parameter for the very small doping limit is of interest to us here. Hence our discussion is concerned with (1) the limit $t>J$ for our perturbation method to be valid, and (2) $t \delta$ (the coherent holon bandwidth) $\ll J$ for the above simplification to hold. The latter inequality allows us to replace the spinon energy $\omega_{p}$ by its value in the undoped limit so that

$$
V_{t o t} \approx \frac{J}{2} \Delta_{0}^{2} \sum_{p} \frac{\gamma_{c}(p)^{2} \gamma_{s}(p)^{2}}{\left(\mu_{B}^{2}-\Delta_{0}^{2} \gamma_{s}(p)^{2}\right)^{3 / 2}} \operatorname{coth}\left(\beta \omega_{p} / 2\right)
$$

where $\mu_{B}$ (the boson chemical potential) varies on a scale set by the antiferromagnetic order parameter $\Delta_{0}$.

Next we examine the behaviour of this interaction in the low-temperature limit remembering the mean field results $[11,12]$. At zero temperature the boson energy gap vanishes at those points where $\Delta_{k}$ takes on its largest values i.e. at $(\pi / 2, \pi / 2)$. At finite temperatures the boson chemical potential is reduced below its zero-temperature value slightly and the dominant contribution to the momentum integration comes from near $(\pi / 2, \pi / 2)$. Cutting off the momentum sum at a value of the order of the zone width yields

$$
V_{e f f}=\left(4 T t^{2} / \Delta_{0}^{2} J^{2} \pi\right) \int_{0}^{q_{c}} q^{3} \mathrm{~d} q /\left(q^{2}+\xi^{2}\right)^{2}
$$

(where $\xi$ denotes the correlation length) which may be considerably simplified when one remembers the form of the gap equation at low temperatures, which after similar manipulations takes the form $1=\left(4 T / \pi J \Delta^{2}\right) \int_{0}^{q_{c}} \mathrm{~d} q q /\left(q^{2}+\xi^{2}\right)$. This leads, to leading logarithmic order, to a very simple result for the effective interaction at low temperatures, 
that is, that $V_{e f f}=t^{2} / J$ apart from corrections of order $T / J$ (which we assume to be small).

The reason for the holon-holon interaction assuming this form may be understood as follows: at long wavelengths and low temperatures the boson pair exchange graphs are dominated by the same low-energy spinon singlet fluctuations that determine the antiferromagnetic order parameter. These fluctuations occur on an energy scale given by $J$, but couple to the holons via the bare hopping matrix element $t$. Hence even the most straightforward perturbation arguments would lead one to expect an interaction of order $t^{2} / J$. We note that it is the bare hopping matrix element $t$ that appears here rather than the renormalized matrix element $t \chi$-in fact the reappearance of such bare energy scales is a standard feature of loop corrections around mean field theories.

Turning to the hole propagators we note that since the temperature scale characterizing the formation of the spiral state is of order $J$, and the coherent holon bandwidth is of order $t \delta$, there exists quite a range of temperatures between these two values. For small hole dopings in which the holon Fermi surface may be considered parabolic, and therefore with a constant density of states $D^{*}(0)$, the zero-frequency, zero-energy Lindhard function is simply approximated as

$$
\chi(0,0)=\sum_{k}\left(-\partial f / \partial \epsilon_{k}\right)=D^{*}(0)\left[1-\exp \left(-\delta / T D^{*} *(0)\right)\right]
$$

where we have used the well known relation for the chemical potential in two dimensions to eliminate the chemical potential. Thus for temperatures higher than the coherent holon bandwidth, $T \gg t \delta, \chi=\delta / T$ (consistent with a Curie susceptibility) so the condition for a long-wavelength charge instability becomes

$$
t^{2} \delta /(J T)=1
$$

which implies long-wavelength charge ordering at a temperature $T_{c}=t^{2} \delta / J$. At zero temperature $\chi(0,0)=1 / D^{*}(0)=1 / t \delta$ so the compressibility $\chi=-J \delta / T$ which is indeed negative.

\section{Discussion and conclusion}

Thus we obtain the result that the compressibility (being the static, long-wavelength limit of the charge density correlation function) diverges at a temperature given by $T_{c}=t(t \delta / J)$, which can be an order of magnitude smaller than the bare electron bandwidth. This suggests that the small-doping sector of the $t-J$ model is unstable against long-wavelength charge ordering. It certainly has been argued that for both small $J / t$ and large $J / t$ the energy cost to add holes is lower for a 'phase separated' state [3] where the holes and spins reside in separate regions of space. The original arguments in favour of phase separation in the small- $J / t$ regime follow from consideration of the frustrated nature of holon motion in an antiferromagnet. The energy cost of putting the hole in a segregated hole-rich region is lower than the cost of placing the hole in a uniform phase. Our result supports this condition as long as we identify the divergence of the static long-wavelength charge susceptibility i.e. the compressibility, as indicating phase separation.

In fact the present work is certainly not the first calculation to find phase separation in the slave fermion $t-J$ model. Auerbach and Larson [20] and Ivanov [21] showed that the ground state energy calculated at the mean field level showed, as a function of doping, a region with a concave dependence on $\delta$ implying a negative compressibility and hence an instability towards phase separation. Later work [22] included the nearest-neighbour 
Coulomb repulsion, treating the model again at mean field level, and found the spiral state to be stable for dopings larger than a critical value. A negative compressibility was also obtained in the large- $S$ limit where the spins behave classically [23]. While these earlier calculations start specifically from the static mean field zero-temperature limit, our calculation provides support for the instability of the spiral state from the standpoint of dynamic finite-temperature change fluctuations with loop corrections.

We should note that the present work is not the only study of fluctuations around the mean field level. The authors of [17] in fact calculated the fermion lifetime resulting from a fermion interaction similar to $V_{\text {eff }}$ in (26). However the first calculation of holon interactions was carried out by Ivanov [24] who constructed the holon-holon Hamiltonian by performing a canonical transformation on the kinetic part of the $t-J$ model (written in terms of Bogoliubov operators for the spiral spin state). Ivanov found the interaction (at $T=0$ ) to be attractive, which he took as signifying a tendency towards p-wave pairing. In fact his conclusions of an attractive interaction are entirely consistent with ours (which imply a negative Landau parameter $F_{0}^{s}$ ).

Similar conclusions regarding the instability of magnetically correlated phases of the $t-J$ model against phase separation were reached using the slave boson approach [7]. These authors studied the stability of the much vaunted dimer and flux phases at the mean level and found that for fixed $J / t$ the magnetically correlated phases were stable at low hole doping while a uniform Fermi liquid phase was stable at higher hole doping. However these authors also found, on examining the free energy as a function of hole doping, regions of negative curvature for fillings away from the insulating value. Via a Maxwell construction they showed the consequent existence of phase separation for hole dopings less than $J / t$, which is certainly consistent with our result of phase separation for $\delta \ll J / t$. In slave boson methods however the occurence of phase separation in this small- $J / t$ region arises again from the frustrated kinetic energy of holes in a background with magnetic correlations. Other extensions of the slave boson approach to models with a greater number of bands [8] find phase separation to occur in the limit where the charge transfer energy is larger than the hopping rate. Phase separation is also suggested in 'weak-coupling' approaches to the Hubbard model, where it is found [25] that the antiferromagnetic Hartree-Fock solution is unstable for infinitesimal hole doping.

The techniques employed in this paper leave room for a lot of improvement-the coherent part of the holon propagator could be corrected to one loop order by including self-energy corrections. Such a correction might incorporate polaronic or 'spin-bag' effects to an appropriate degree, lowering the holon energy as well as changing the quasiparticle weight of the coherent holon propagator. A more thorough 'mode-coupling' diagrammatic analysis might allow for the charge stabilities to occur at wavevectors other than the $q=0$ limit studied in this paper. According to the present method we obtain such a strong holon-holon interaction via the exchange of spin fluctuations that the most likely scenario for phase separation is by the complete segregation of charge and spin (as in the earlier methods $[3,7,8])$, thus enabling the holons to avoid the exchange of spin fluctuations.

In conclusion, we have, in this paper, studied the charge susceptibility within the framework of the slave fermion formulation of the $t-J$ model in which magnetic correlations are naturally incorporated at the lowest order. We find that at the mean field level the equaltime density correlation function agrees qualitatively at least with the conclusions of recent high-temperature series studies. Going beyond the mean field level and including loop corrections via the ladder and bubble series suggests, for small doping $(\delta \ll J / t)$ at least, an instability against charge ordering at long wavelength which we interpret as indicating the onset of phase separation. Thus instability is driven by antiferromagnetic spin fluctuations 
which couple to the holons via the bare hopping matrix element $t$, leading to a large effective holon-holon interaction of order $t^{2} / J$. This interaction then enters the ladder and bubble series for the charge susceptibility. The results therefore complement previous mean field studies by obtaining evidence for phase separation from the loop corrections themselves. Extensions to more realistic models with a greater number of bands is left to future study.

\section{References}

[1] Anderson P W 1990 Phys. Rev. Lett. 652306

[2] Puttika W O et al 1994 Phys. Rev. Lett. 73170

[3] Emery V J, Kivelson S A and Lin H Q 1990 Phys. Rev. Lett. 64475

[4] Marder M, Papanicolau N and Psaltakis G C 1990 Phys. Rev. B 416920

[5] Muller K A and Benedek G (ed) 1992 Phase Separation in Cuprate Superconductors (Erice, 1992)

[6] Read N and Newns D M 1988 Adv. Phys. 36999

[7] Grilli M, Castellani C and Kotliar G 1992 Phys. Rev. B 4510805

[8] Castellani C, Grilli M and Kotliar G 1991 Phys. Rev. B 438000

Caprara S and Grilli M 1994 Phys. Rev. B 496971

[9] Rasul J W 1995 Phys. Rev. B 512632

[10] Arovas D and Auerbach A 1988 Phys. Rev. B 38316

[11] Yoshioka D 1989 J. Phys. Soc. Japan 581516

[12] Flensberg K, Hedergaard P and Pederson M B 1989 Phys. Rev. B 40850

[13] Jayaprakash C, Krishnamurthy H R and Sarker S 1989 Phys. Rev. B 402610

[14] Krishnamurthy H R et al Proc. Santa Fe Conf. on Strongly Correlated Electrons (Santa Fe)

[15] Sarker S 1992 Phys. Rev. B 468616

[16] Boies D, Jackson A and Tremblay A-M S Preprint

[17] Li Y M, Sheng D N, Su Z B and Yu L 1992 Phys. Rev. B 455428 Bai Yujong 1994 PhD Thesis University of Michigan

[18] Tohyama T, Horsch P and Maekawa S 1995 Phys. Rev. Lett. 74980

[19] Levin K and Valls O 1979 Phys. Rep. 981

[20] Auerbach A and Larson B 1991 Phys. Rev. B 437800

[21] Ivanov T I 1991 Phys. Rev. B 4412077

[22] Sarker S 1993 Phys. Rev. B 472940

[23] Mori H and Hamada M 1993 Phys. Rev. B 486242

[24] Ivanov T I 1990 Phys. Lett. 145A 201

[25] Singh A, Tesanovich Z and Kim Ju 1991 Phys. Rev. B 447757 